\title{
Antimalarial Drug Resistance Surveillance in China, 2016-2020
}

\author{
Fang Huang ${ }^{1, *}$; Hui Liu ${ }^{2}$; He Yan ${ }^{1}$; Shuisen Zhou ${ }^{1}$; Zhigui Xia ${ }^{1}$
}

\begin{abstract}
Introduction: Antimalarial drug resistance, especially artemisinin resistance, has been a global threat to reduce morbidity and mortality and to eliminate malaria. Antimalarial drug resistance surveillance could provide evidence for the efficacy of a national drug policy for treatment of all reported malaria cases.

Methods: The therapeutic efficacy of dihydroartemisinin-piperaquine (DHA-PPQ) and chloroquine (CQ) for the treatment of uncomplicated Plasmodium falciparum and Plasmodium vivax infection, respectively, was evaluated by enrolling patients between 2016 and 2020. Surveillance of molecular markers involved collecting blood samples by passive or active detection and amplifying the drug resistance genes Pfcrt, Pfmdrl, Pfdhfr, Pfdhps, and PfK13.
\end{abstract}

Results: DHA-PPQ and CQ were effective for the treatment of $P$. falciparum and $P$. vivax infections, with cure rates $>90 \%$ and $100 \%$, respectively. Among the 2,492 samples, the Pfort wild-type CVMNK was the most frequent haplotype $(70.1 \%, 1,747 / 2,492)$, while $19.4 \%(484 / 2,492)$ displayed the triple mutant haplotype CVIET. In total of 228 isolates with sequencing in Pfdhfr and Pfdhps, high mutant prevalence of $98.7 \%$ and $95.2 \%$ were detected, respectively. A total of 54 non-synonymous mutations in the PfK13 propeller domain were confirmed with a prevalence of $3.5 \%(87 / 2,483)$, and the most common mutation was A578S, with a proportion of $16.1 \%$ (14/87), followed by Q613E (6.9\%, 6/87).

Conclusion and Implications for Public Health Practice: DHA-PPQ and CQ, the first line drugs for $P$. falciparum and $P$. vivax treatment, showed efficacy in China. Molecular markers showed high levels of polymorphism and resistance, which revealed an early warning of drug resistance in imported cases.

\section{INTRODUCTION}

Great progress on malaria elimination has been achieved in China, and thousands of imported malaria cases annually remain a key focus in malaria elimination and the post-elimination phase in China (1-2). In 2012, China established the malaria diagnosis reference laboratory network focusing on malaria diagnosis, treatment, and surveillance, which covered national, provincial, and county levels (3). According to the World Health Organization (WHO) malaria surveillance reference manual in the elimination stage, the antimalarial drug resistance surveillance network in China was established in 2016 and covered 8 provincial-level administrative divisions (PLADs) with more imported malaria cases or a high risk of malaria re-transmission including the following: Yunnan, Henan, Zhejiang, Shandong, Shanghai, Hunan, Sichuan, and Guangxi. This network depended on the malaria diagnosis reference laboratories and the Chinese Infectious Disease Reporting System (CIDRS), the largest web-based communicable diseases reporting system to provide diagnostic and treatment support and case information for drug resistance surveillance (2).

Up to now, $P$. falciparum has developed resistance to nearly all antimalarial drugs, including artemisinin and its derivatives (4). Antimalarial resistance surveillance is designed to monitor the efficacy against any Plasmodium spp. of the recommended first and second line drugs, and any new medicine or drug that is to be evaluated before being introduced into the national drug policy (5). Therapeutic efficacy studies (TES) remain the gold standard for assessing the efficacy of antimalarial drug, and integrated drug efficacy studies (iDES) will be introduced in low malaria transmission or malaria elimination areas, where surveillance capacity is strong enough to ensure all the data on malaria cases can be used to generate information on drug efficacy. Currently, molecular markers involved in $P$. falciparum resistance against antimalarial drugs have been confirmed (Table 1): chloroquine (CQ) resistance markers of $P$. falciparum CQ-resistance transporter $(P f c r t)$, multidrug resistance gene of $P$. falciparum multidrug resistance gene 1 (Pfmdrl), and $P$. 
TABLE 1. Haplotypes of Pfcrt, Pfmdr1, Pfdhfr, and Pfdhps genes conferring resistance to antimalarial drugs in Plasmodium falciparum isolates.

\begin{tabular}{|c|c|c|c|c|c|c|}
\hline Genes & Regions and countries ( $n$ ) & Haplotype & Number & Prevalence & Drug & Chemical family \\
\hline \multirow{5}{*}{ Pfcrt $(n=2,492)$} & \multirow{5}{*}{$\begin{array}{c}\text { Eastern Africa (145), } \\
\text { Western Africa (957), } \\
\text { Central Africa (733), } \\
\text { Southern Africa (619), } \\
\text { North Africa (2), } \\
\text { Other regions (36) }\end{array}$} & Wild type (CVMNK) & 1,747 & $70.1 \%$ & \multirow{5}{*}{ Chloroquine } & \multirow{5}{*}{ 4-Aminoquinolines } \\
\hline & & CVIET & 484 & $19.4 \%$ & & \\
\hline & & CVMNI & 120 & $4.8 \%$ & & \\
\hline & & $\mathrm{CV} \mathrm{M} / \underline{\underline{I}} \mathrm{~N} / \underline{\mathrm{E}} \mathrm{K} / \underline{\mathbf{T}}$ & 74 & $3.0 \%$ & & \\
\hline & & Others ${ }^{*}$ & 67 & $2.7 \%$ & & \\
\hline \multirow{6}{*}{ Pfmdr1 $(\mathrm{n}=617)$} & \multirow{6}{*}{$\begin{array}{l}\text { Eastern Africa (20), } \\
\text { Western Africa (185), } \\
\text { Central Africa (165), } \\
\text { Southern Africa (247) }\end{array}$} & Wild type (NYD) & 307 & $49.8 \%$ & \multirow{6}{*}{$\begin{array}{l}\text { Amodiaquine } \\
\text { Lumefantrine }\end{array}$} & \multirow{6}{*}{$\begin{array}{l}\text { 4-Aminoquinolines } \\
\text { Amino-alcohols }\end{array}$} \\
\hline & & NED & 124 & $20.1 \%$ & & \\
\hline & & $\underline{\text { YFD }}$ & 82 & $13.3 \%$ & & \\
\hline & & N Y/E D & 42 & $6.8 \%$ & & \\
\hline & & YYD & 31 & $5.0 \%$ & & \\
\hline & & Others $^{\dagger}$ & 31 & $5.0 \%$ & & \\
\hline \multirow{4}{*}{$\operatorname{Pfdhfr}(\mathrm{n}=228)$} & \multirow{4}{*}{$\begin{array}{c}\text { Angola (158), } \\
\text { Equatorial Guinea (70) }\end{array}$} & Wild type (ANCSI) & 3 & $1.3 \%$ & \multirow{4}{*}{ Pyrimethamine } & \multirow{4}{*}{ Antifolates } \\
\hline & & AIRNI & 160 & $70.2 \%$ & & \\
\hline & & AICNI & 55 & $24.1 \%$ & & \\
\hline & & Others ${ }^{\S}$ & 10 & $4.4 \%$ & & \\
\hline \multirow{5}{*}{ Pfdhps $(\mathrm{n}=228)$} & \multirow{5}{*}{$\begin{array}{c}\text { Angola (158), } \\
\text { Equatorial Guinea (70) }\end{array}$} & Wild type (SAKAA) & 11 & $4.8 \%$ & \multirow{5}{*}{ Sulfadoxine } & \multirow{5}{*}{ Antifolates } \\
\hline & & 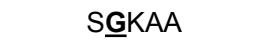 & 153 & $67.1 \%$ & & \\
\hline & & S $\underline{G E A A}$ & 25 & $11.0 \%$ & & \\
\hline & & $\underline{A G K A A}$ & 24 & $10.5 \%$ & & \\
\hline & & Others ${ }^{\pi}$ & 15 & $6.6 \%$ & & \\
\hline
\end{tabular}

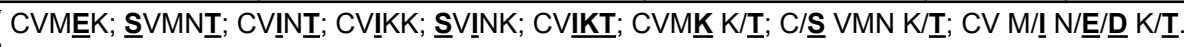

${ }^{\dagger} \underline{Y} Y \underline{Y} ; N \underline{F Y} ; N / \underline{Y} \mathbf{F D} ; N / \underline{Y} Y D ; \underline{Y} Y / \underline{F} D ; N / \underline{Y} Y / \underline{F} D ; N / \underline{Y} \underline{Y} D / \underline{Y}$.

$\S$ ANRNI; AIRNI; AIRNL.

"AAKAA.

falciparum dihydrofolate reductase (Pfdhfr) and $P$. falciparum dihydropteroate synthase (Pfdhps) conferring resistance to pyrimethamine and sulfadoxine (SP), respectively. Point mutations in PfK13 propeller region have been confirmed to be associated with artemisinin resistance (6). Since 2008, TES and molecular markers surveillance, 2 primary tools for monitoring antimalarial drug resistance, have been implemented in China for several years (7-9). The aim of this study was to investigate the efficacy of first line drugs for malaria treatment in Yunnan Province and characterize the drug resistance genes in the imported $P$. falciparum isolates from 2016 to 2020 in order to produce a systematic picture of antimalarial resistance to guide drug policy.

\section{METHODS}

TES and iDES were conducted to evaluate dihydroartemisinin-piperaquine (DHA-PPQ) for $P$. falciparum treatment and CQ for $P$. vivax treatment in
Yunnan Province between 2016 and 2020. Patients with malaria infection, including symptomatic and asymptomatic cases confirmed in a malaria reference laboratory by passive or active case detection, were included, with the exception of patients with severe malaria who were also treated with other medicines for severe complications. Patients were followed-up from the first day of treatment (Day 0) to the specified last day of the follow-up period when appropriate for the infecting species was identified and the treatment was administered (Figure 1). Treatment outcome was determined following the WHO guidelines for therapeutic efficacy monitoring including early treatment failure (ETF), late clinical failure (LCF), late parasitological failure (LPF), and adequate clinical and parasitological response (ACPR) (5).

The haplotypes and polymorphisms of Pfcrt, Pfmdr1, Pfdhfr, Pfdhps, and PfK13 (propeller domain) were genotyped by sequencing the samples collected from imported malaria cases from different sub-regions of Africa, Asia, and Oceania between 2012 and 2019. 


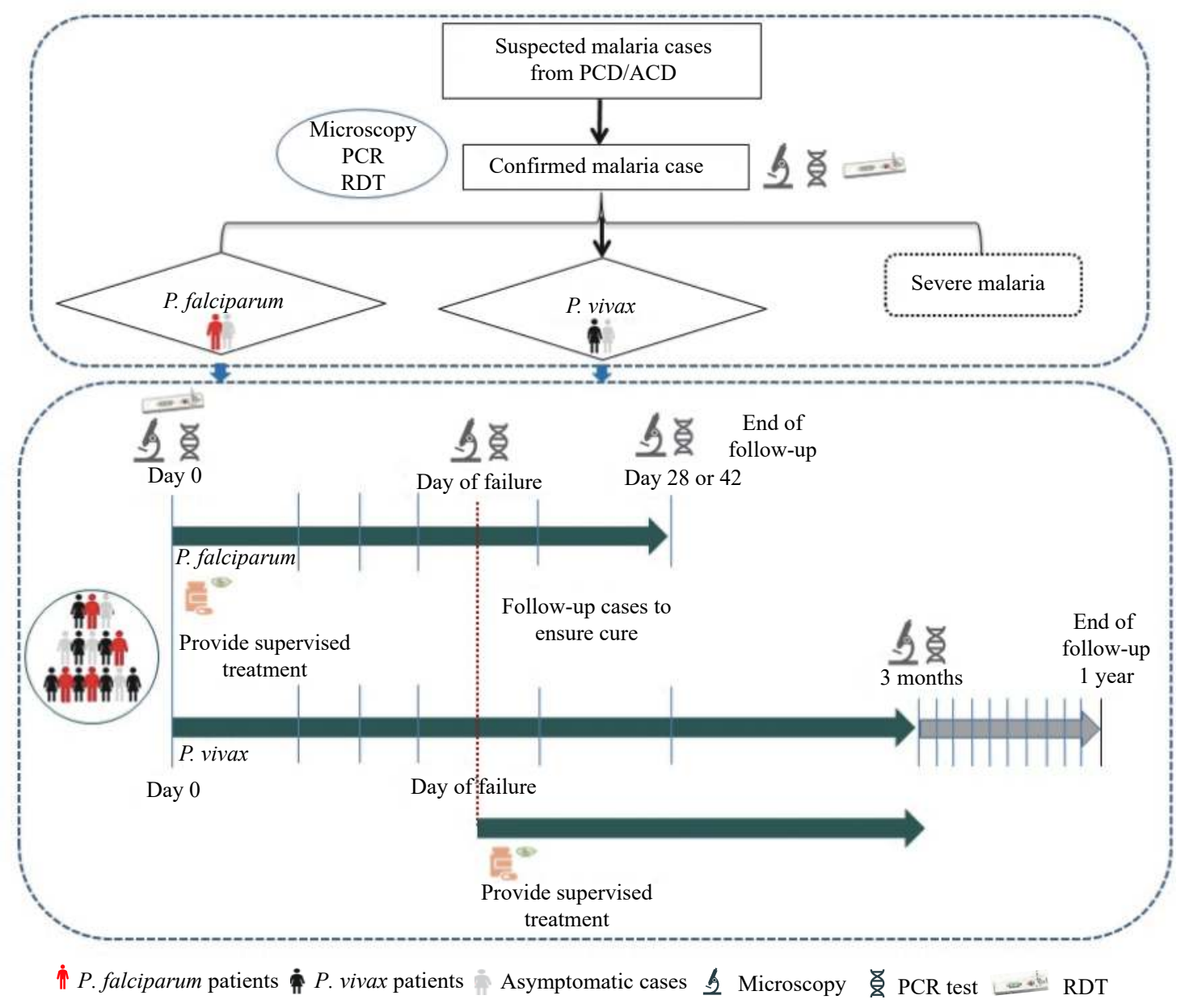

FIGURE 1. Flowchart of iDES in case detection, treatment, and follow-up integration in the routine malaria surveillance system in China between 2016-2020.

Abbreviations: $P C D=$ passive case detection; $A C D=$ active case detection; $P C R=$ polymerase chain reaction; RDT=rapid diagnosis test.

The sequence data were aligned and compared with the reference sequences from PlasmoDB (http://www. plasmodb.org).

\section{RESULTS}

A total of 60 participants were enrolled and received observed treatment with DHA-PPQ or CQ between 2016 and 2020. The outcome of DHA-PPQ efficacy was adjusted and corrected using a nested polymerase chain reaction (PCR) assay. Overall, 39 participants with $P$. falciparum infection completed the 42 days of follow-up and 1 was lost to follow-up. The average ACPR rate for DHA-PPQ in 2016 (9/10), 2017 $(15 / 16)$, and $2018(12 / 13)$ was $92.0 \%$ (minimum: 90.0\%; maximum: 93.8\%) (Table 1). In 2017, 1 patient showed recurrent parasite infection after DHAPPQ treatment and was originally determined as treatment failure but corrected to $P$. vivax infection by
PCR amplification. In addition, 3 out of 39 patients (7.7\%) were still positive on Day 3 by microscopy detection, which indicated that the time of parasite clearance was delayed and was an early warning of antimalarial drug resistance. The iDES was initiated in Yunnan Province in 2020. A total of 19 imported $P$. vivax patients were recruited and finished the 28 days of follow-up. Among them, 2 participants were lost and end of follow-up. The cure rate of CQ for $P$. vivax treatment in Yunnan Province was 100.0\% (17/17).

$P f c r t$ gene was sequenced in 2,492 $P$. falciparum isolates imported from Africa. The most prevalent haplotype was the wild type $(70.1 \%, 1,747 / 2,492)$. A total of 8 mutant haplotypes including single, double, and triple mutant types were detected, and another 4 mixed mutant haplotypes were observed simultaneously (Table 2 ). The triple mutant haplotype CVIET $(19.4 \%, 484 / 2,492)$ was the most common mutant type, followed by the single mutant haplotype 
TABLE 2. Treatment outcome of therapeutic efficacy studies (TES) and integrated drug efficacy studies in Yunnan Province, 2016-2020.

\begin{tabular}{|c|c|c|c|c|c|}
\hline \multirow{2}{*}{ Item } & \multicolumn{3}{|c|}{ TES of DHA-PIP for $P$. falciparum treatment } & \multirow{2}{*}{$\begin{array}{c}\text { iDES of CQ for } P \text {. vivax treatment } \\
2020\end{array}$} & \multirow{2}{*}{ Total } \\
\hline & 2016 & 2017 & 2018 & & \\
\hline ETF & 1 & $2^{*}$ & 1 & 0 & 4 \\
\hline LCF & 0 & 0 & 0 & 0 & 0 \\
\hline LPF & 0 & 0 & 0 & 0 & 0 \\
\hline ACPR & 9 & 14 & 12 & 19 & 55 \\
\hline PCR-corrected ACPR & 9 & 15 & 12 & 19 & 56 \\
\hline LFU * & 0 & 0 & 1 & 2 & 3 \\
\hline Total & 10 & 16 & 13 & 21 & 60 \\
\hline No. of Day3 (+) & 0 & 3 & 1 & NA & \\
\hline
\end{tabular}

Abbreviations: $\mathrm{ETF}=$ early treatment failure; $\mathrm{LCF}=$ late clinical failure; $\mathrm{LPF}=$ late parasitological failure; $\mathrm{ACPR}=\mathrm{adequate}$ clinical and parasitological response; $L F U=$ lost to follow-up. $D H A-P P Q=$ dihydroartemisinin-piperaquine; $C Q=$ chloroquine; $N A=$ not available.

One patient in 2017 who showed treatment failure after DHA-PPQ treatment was confirmed to have $P$. vivax infection, using PCR amplification, and the outcome was changed to PCR-corrected ACPR.

CVMN $\underline{T}(4.8 \%, 120 / 2,492)$.

There were 617 P. falciparum isolates collected from Southern Africa $(\mathrm{n}=247)$, Western Africa $(\mathrm{n}=185)$, Central Africa $(\mathrm{n}=165)$, and Eastern Africa $(\mathrm{n}=20)$ for Pfmdr1 gene sequencing. The Pfmdr1 wild type NYD was common $(49.8 \%$, 307/617). Point mutations were identified in codons 86 and 184, and the D1246Y mutation was absent. In addition, 2 single and 3 double mutant haplotypes were identified. The single mutant haplotype NED was the highest prevalent mutation $(20.1 \%$, 124/617). The double mutant haplotype YFD $(13.3 \%, 82 / 617)$ was more common than the haplotypes of $\underline{Y} \underline{Y}(0.5 \%, 3 / 617)$ and $\mathrm{NFY}$ $(0.2 \%, 1 / 617)$. The mixed mutant haplotypes were detected. Among them, N Y/F D was the main one and detected in 42 isolates (Table 2).

Pfdhfr and Pfdhps were genotyped in $70 P$. falciparum samples from Equatorial Guinea and 158 from Angola. Most of them revealed high mutant prevalence and only 3 contained the Pfdhfr wild type ANCSI (Table 2). A total of 5 mutant haplotypes were identified and the most prevalent was the triple mutant haplotype AIRNI $(70.2 \%, 160 / 228)$, followed by AICNI $(24.1 \%, 55 / 228)$. No mixed mutant haplotype in the Pfdhfr gene. There were 8 isolates in Pfdhps wild type SAKAA $(4.8 \%, 11 / 228)$. In addition, 1 single mutant (AAKAA), 2 double mutants (SGEAA and AGKAA), 2 triple mutants (AGKA $\underline{\mathbf{S}}$ and SGEGA), and 1 mixed mutant haplotype were identified. The haplotype SGKAA with single mutant was common, with a prevalence of $67.1 \%(153 / 228)$, while the haplotypes SGEAA $(11.0 \%, 25 / 228)$ and AGKAA $(10.5 \%, 24 / 228)$ with double mutant were relatively less prevalent.
A total of 2,483 samples were collected including 941 from western Africa, 746 from central Africa, 666 from southern Africa, 120 from eastern Africa, 4 from northern Africa, and 6 from Asia and Oceania. Of all the successful sequencing samples, 2,344 isolates were wild type, 87 isolates carried $P f K 13$ non-synonymous (NS) mutations, and 26 synonymous mutations were detected in 52 samples (Table 3). The mutant alleles A578S was detected in 14 isolates $(0.6 \%, 14 / 2,483)$, which was the most frequent $P f K 13$ mutation. Q613E variant was carried by 6 samples, which was the second-most frequent mutation; $74.1 \%(40 / 54)$ and $65.4 \%(17 / 26)$ of the NS and synonymous mutations were identified in one sample each. There were 11 samples carried PfK13 mutations associated with artemisinin resistance consisting of $\mathrm{C} 580 \mathrm{Y}, \mathrm{R} 561 \mathrm{H}$, R539T, M476I, and P553L. C469C variants were detected in 10 isolates with the highest prevalence of synonymous mutation.

\section{DISCUSSION}

Antimalarial drug resistance is an obstacle for malaria elimination worldwide, especially as the emergence of artemisinin resistance in the Greater Mekong Subregion has been an urgent regional public health concern (10-11). In this study, DHA-PPQ was efficacious with a high cure rate in Yunnan Province, although it has been used as the first-line drug for the treatment of $P$. falciparum for more than 10 years in China. P. vivax resistance to CQ has also been identified in Southeast Asia and part of Africa (12). A total of 17 imported $P$. vivax patients fully completed the follow up in iDES with $100 \%$ efficacy. According 
TABLE 3. Numbers and proportion of non-synonymous and synonymous mutations in the PfK 13 gene detected in imported $P$. falciparum isolates collected during 2012-2019.

\begin{tabular}{|c|c|c|c|c|c|}
\hline \multicolumn{3}{|c|}{ Non-synonymous mutations in PfK13 } & \multicolumn{3}{|c|}{ Synonymous mutations in PfK13 } \\
\hline Variant & $\mathbf{n}$ & Proportion & Variant & $\mathbf{n}$ & Proportion \\
\hline A578S & 14 & $16.1 \%$ & C469C & 10 & $19.2 \%$ \\
\hline Q613E & 6 & $6.9 \%$ & $\mathrm{R} 471 \mathrm{R}$ & 6 & $11.5 \%$ \\
\hline C580Y & 3 & $3.4 \%$ & V589V & 4 & $7.7 \%$ \\
\hline M579I & 3 & $3.4 \%$ & A676A & 3 & $5.8 \%$ \\
\hline N664D & 3 & $3.4 \%$ & T478T & 3 & $5.8 \%$ \\
\hline $\mathrm{C} 580 \mathrm{~F}$ & 2 & $2.3 \%$ & Y493Y & 3 & $5.8 \%$ \\
\hline D464N & 2 & $2.3 \%$ & A627A & 2 & $3.8 \%$ \\
\hline N694K & 2 & $2.3 \%$ & G496G & 2 & $3.8 \%$ \\
\hline P441S & 2 & $2.3 \%$ & N664N & 2 & $3.8 \%$ \\
\hline R539T & 2 & $2.3 \%$ & Others $^{\dagger}$ & 17 & $32.7 \%$ \\
\hline R561H & 2 & $2.3 \%$ & & & \\
\hline R575T & 2 & $2.3 \%$ & & & \\
\hline R622I & 2 & $2.3 \%$ & & & \\
\hline V589I & 2 & $2.3 \%$ & & & \\
\hline Others ${ }^{*}$ & 40 & $46.0 \%$ & & & \\
\hline Total & 87 & $100.0 \%$ & & 52 & $100.0 \%$ \\
\hline
\end{tabular}

* Others refer to the other non-synonymous mutations and one isolate of each variant, including A481T, A569T, A578T, A621S, A626T, C469F, C469F, D462N, D464E, D584V, D648N, D648Y, E556K, F434Y, F662C, G665S, I634T, I646K, I683R, K503E, K610R, K658Q, L457S, L488V, L619E, L663V, M476I, M562I, N629S, P443R, P553L, P574L, Q613H, R575K, T437N, T474I, V589A, V603E, V650F, V692L.

${ }^{\dagger}$ Others refer to the other synonymous mutations and one isolate of each variant, including A557A, G454G, G533G, G638G, K610K, L492L, P443P, R417R, R575R, S477S, T535T, T573T, T685T, V487V, V510V, V666V, Y502Y.

to the latest guideline of antimalarial drugs policy in China, the first-line treatment drug for $P$. falciparum and $P$. vivax infections in China were artemisininbased combination therapies (ACTs) and CQ plus primaquine, respectively. Although there was no evidence for DHA-PPQ and CQ resistance emerging in China, continued antimalarial drug resistance surveillance focus on the imported cases by designated public hospitals at the provincial level should be strengthened in the post-elimination stage.

Drug resistance is the main cause of clinical treatment failure. Therefore, characterization of the drug resistance markers is an important way to reveal the drug resistance and treatment failure. In the recent 20 years, the CQ resistance of $P$. facliparum decreased along with the wild type of $P f c r t$ increasing caused by the termination of the use of CQ when its resistance spread widely in Africa in the 1990s (13). In this study, the Pfcrt wild type was the most common haplotype, and the mutant prevalence was rising. Pfmdr1 mutations were observed in codons 86 and 184 in this study, although the wild type (49.8\%) was the majority. SP resistance genes Pfdhfr and Pfdhps displayed high mutant prevalence of $98.7 \%$ and $95.2 \%$, respectively. SP, the only choice for the intermittent preventive treatment in infants (IPTi) remain in use in Africa. This study indicated most of the imported $P$. falciparum cases in China showed high-level SP resistance. High polymorphisms of PfK13 propeller region were revealed and 54 nonsynonymous mutations and 26 synonymous mutations were detected, with low prevalence of $3.5 \%$ and $2.1 \%$, respectively. The A578S mutation was detected in 14 isolates, which was the most frequent mutation in the $P f K 13$ propeller domain in the isolates from Africa (14). A few patients carrying PfK13 mutations associated with artemisinin resistance still showed sensitivity to antimalarials based on the individual information collected from CIDRS, but the systematic efficacy study data were absent. However, the risk of the importation of artemisinin resistance should be particularly concerning.

The study was subject to at least two limitations. First, TES and iDES were only implemented in Yunnan Province and the sample size was small. Further study will be extensively rolled out in the eight 
key provinces and then nationwide in the future. Second, plasmepsin IIIIII (pm2/3), another molecular marker associated with PPQ resistance (15), the partner drug of DHA, was not involved in this study.

Acknowledgements: Staff members of the provincial CDC involved in the national antimalarial drug resistance network; all the malaria patients recruited in TES/iDES; and the participants who provided blood samples for this study.

Conflicts of interest: No competing interests.

Funding: The Natural Science Foundation of Shanghai (No. 18ZR1443400), the Fifth Round of Three-Year Public Health Action Plan of Shanghai (No. GWV-10.1-XK13), and the National Important Scientific \& Technological Project (2018ZX10101 002-002).

doi: $10.46234 / \mathrm{ccdcw} 2021.099$

\# Corresponding author: Fang Huang, huangfang@nipd.chinacdc.cn.

\begin{abstract}
${ }^{1}$ National Institute of Parasitic Diseases, Chinese Center for Disease Control and Prevention, Chinese Center for Tropical Diseases Research, WHO Collaborating Center for Tropical Diseases, National Centre for International Research on Tropical Diseases, NHC Key Laboratory of Parasite and Vector Biology (National Institute of Parasitic Diseases, Chinese Center for Disease Control and Prevention), Shanghai, China; ${ }^{2}$ Yunnan Institute of Parasitic Diseases, Puer, China.
\end{abstract}

Submitted: March 16, 2021; Accepted: April 20, 2021

\section{REFERENCES}

1. Feng XY, Levens J, Zhou XN. Protecting the gains of malaria elimination in China. Infect Dis Poverty 2020;9(1):43. http://dx.doi.org/ 10.1186/s40249-020-00661-y.

2. Zhang L, Feng J, Zhang SS, Xia ZG, Zhou SS. The progress of national malaria elimination and epidemiological characteristics of malaria in China in 2017. Chin J Parasitol Parasit Dis 2018; 36(3): 201-9. https://d.wanfangdata.com.cn/periodical/zgjscxyjscbzz201803001. (In Chinese).

3. Yin JH, Yan H, Huang F, Li M, Xiao HH, Zhou SS, et al. Establishing a China malaria diagnosis reference laboratory network for malaria elimination. Malar J 2015;14(1):40. http://dx.doi.org/10.1186/s12936- 015-0556-z

4. White NJ. Antimalarial drug resistance. J Clin Invest 2004;113(8):1084 - 92. http://dx.doi.org/10.1172/JCI21682.

5. WHO. Methods for surveillance of antimalarial drug efficacy. Geneva: World Health Organization. 2009. https://www.who.int/malaria/ publications/atoz/9789241597531/en/.

6. WHO. Malaria surveillance, monitoring \& evaluation: a reference manual. Geneva: World Health Organization. 2009. https://www. who.int/malaria/publications/atoz/9789241565578/en/.

7. Huang F, Tang LH, Yang HL, Zhou SS, Sun XD, Liu H. Therapeutic efficacy of artesunate in the treatment of uncomplicated Plasmodium falciparum malaria and anti-malarial, drug-resistance marker polymorphisms in populations near the China-Myanmar border. Malar J 2012;11(1):278. http://dx.doi.org/10.1186/1475-2875-11-243.

8. Huang F, Tang L, Yang H, Zhou S, Liu H, Li J, Guo S. Molecular epidemiology of drug resistance markers of Plasmodium falciparum in Yunnan Province, China. Malar J 2012;11(1):243. http://dx.doi.org/ 10.1186/1475-2875-11-278.

9. Liu H, Yang HL, Tang LH, Li XL, Huang F, Wang JZ, et al. In vivo monitoring of dihydroartemisinin-piperaquine sensitivity in Plasmodium falciparum along the China-Myanmar border of Yunnan Province, China from 2007 to 2013. Malar J 2015;14(1):47. http://dx.doi.org/10.1186/s12936-015-0584-8.

10. van der Pluijm RW, Imwong M, Chau NH, Hoa NT, Thuy-Nhien NT, Thanh NV, et al. Determinants of dihydroartemisinin-piperaquine treatment failure in Plasmodium falciparum malaria in Cambodia, Thailand, and Vietnam: a prospective clinical, pharmacological, and genetic study. Lancet Infect Dis 2019;19(9):952 - 961. http://dx. doi.org/10.1016/S1473-3099(19)30391-3.

11. WHO. Artemisinin resistance and artemisinin-based combination therapy efficacy. Geneva: World Health Organization; 2018. https:// www.who.int/publications/i/item/status-report-on-artemisinin-resistanceand-act-efficacy.

12. Ferreira MU, Nobrega de Sousa T, Rangel GW, Johansen IC, Corder RM, Ladeia-Andrade S, Gil JP. Monitoring Plasmodium vivax resistance to antimalarials: Persisting challenges and future directions. Int J Parasitol Drugs Drug Resist 2021;15:9 - 24. http://dx.doi.org/10. 1016/j.ijpddr.2020.12.001

13. Djimdé A, Doumbo OK, Cortese JF, Kayentao K, Doumbo S, Diourté $\mathrm{Y}$, et al. A molecular marker for chloroquine-resistant falciparum malaria. N Engl J Med 2001;344(4):257 - 63. http://dx.doi.org/10. 1056/NEJM200101253440403.

14. Kamau E, Campino S, Amenga-Etego L, Drury E, Ishengoma D, Johnson K, et al. K13-propeller polymorphisms in Plasmodium falciparum parasites from sub-Saharan Africa. J Infect Dis 2015;211 (8):1352 - 5. http://dx.doi.org/10.1093/infdis/jiu608.

15. Witkowski B, Duru V, Khim N, Ross LS, Saintpierre B, Beghain J, et al. A surrogate marker of piperaquine-resistant Plasmodium falciparum malaria: a phenotype-genotype association study. Lancet Infect Dis 2017;17(2):174 - 83. http://dx.doi.org/10.1016/S1473-3099(16)30 $415-7$. 